\title{
Bilateral vision loss associated with radiofrequency exposure
}

This article was published in the following Dove Press journal:

Clinical Ophthalmology

II December 2012

Number of times this article has been viewed

\author{
Dianna Liu \\ Franz Marie Cruz \\ Prem S Subramanian \\ Wilmer Eye Institute, The Johns \\ Hopkins School of Medicine, \\ Baltimore, Maryland, USA
}

Correspondence: Prem S Subramanian Wilmer Eye Institute, Johns Hopkins Hospital, 600 North Wolfe Street, Baltimore, Maryland 21287 , USA

Tel +l 4109558679

$\mathrm{Fax}+\mathrm{I} 4106149240$

Email psubram I@jhmi.edu
Abstract: A 57-year-old otherwise healthy woman presented with painless binocular vision loss 1 week after direct application of radiofrequency energy to her orbits. She had no light perception bilaterally. Pupils were dilated and not reactive to light. Fundoscopic exam initially showed optic disc swelling in the right eye and a normal-appearing disc in the left eye. Magnetic resonance imaging of the brain and orbits showed gadolinium enhancement of both intraorbital optic nerves. She underwent a course of high-dose steroid treatment without recovery of vision. Optic discs were pale 11 weeks after injury. With exclusion of other possible causes, this represents a unique case of irreversible binocular optic nerve damage and blindness secondary to radiofrequency exposure.

Keywords: optic neuropathy, blindness, radiofrequency, vision loss

Radiofrequency radiation (RFR) is a form of electromagnetic radiation at frequencies ranging from $3 \mathrm{kHz}$ to $300 \mathrm{GHz}$. Several household appliances emit RFR, including the television, radio, microwave oven, and cellular phones. In medicine, RFR is emitted by diathermy equipment, electrocautery devices, magnetic resonance imaging (MRI) scanners, and hyperthermia devices for cancer treatment. RFR causes damage by heating body tissues, producing protein denaturation, cell membrane disruption, and cell death.

Few papers report optic nerve damage as the result of RFR. ${ }^{2-5}$ Monocular blindness resulted as an immediate complication of trigeminal RF rhizotomy for the treatment of trigeminal neuralgia. Visual loss has also been reported following the use of electrocautery probes to achieve hemostasis in orbital and sinus surgeries. ${ }^{4,5}$ Conflicting data exist on the induction of cataract in humans (primarily radar operators) by lowlevel chronic RFR exposure. ${ }^{6}$ We report on a case of binocular blindness from RFR exposure for alternative medical therapy.

\section{Case report}

A 57-year-old Asian female with no significant past medical history presented to the neuro-ophthalmology clinic with visual loss in both eyes. Family medical history was unremarkable. She was a moderate $(-6.00 \mathrm{D}$ OU) myope. About 18 months before presentation, the patient was collecting rocks in stagnant water and soon thereafter developed a sensation of tapeworms in her scalp. She was seen by multiple local dermatologists; no parasites were ever identified. She was diagnosed with delusional parasitosis and was advised psychiatric evaluation. She reported 
seeing white spaghetti strands of worms across her face and hearing the movement of the worms in her ears. She had acquired mebendazole tablets from Hong Kong and had been self-administering the drug on an intermittent basis. She had also tried topical treatments with chemicals, such as turpentine.

When none of these alleviated her symptoms, she acquired a ProGen II 4050 frequency generator (Resonant Light Technology Inc, Courtenay, British Columbia, Canada) for bioelectronic therapy, which claims to kill microorganisms. She applied the contact pads on her scalp several times. Feeling that immature worms were in her eyelids and lashes, she applied the contact pads $(38 \times 88 \mathrm{~mm})$ directly over her eyelids for a single treatment of each eyelid, during which the generator output ranged between 90 to $580 \mathrm{kHz}$ for about 30 seconds at 14 volts. She did not recall the pulse frequency. She had the contact pads in place for 10 minutes on her left and 15 minutes on her right eye. During that time she reported no pain or heat. She felt only the pulsations "beating her eyelids." No pressure was applied to the eyelids to hold the patches in place.

The following day she noted blurred vision in both eyes, which she described as "snowy." She had no pain with eye movement, redness, photosensitivity, or transient visual obscurations. She denied any headache, fever, numbness, weakness, or paresthesia. Over the next week, her vision slowly worsened to complete blindness. She was admitted to a local hospital where computed tomography (CT) of the head showed moderate atrophic changes in both frontal lobes. Orbital CT demonstrated myopic globes without any mass. Magnetic resonance imaging (MRI) of the brain and orbits with gadolinium contrast showed T2 hyperintensity in the right optic nerve (Figure 1). Cranial magnetic resonance (MR) venography was unremarkable. Erythrocyte sedimentation rate (ESR) was $7 \mathrm{~mm} /$ hour and C-reactive protein $(\mathrm{CRP})<0.1 \mathrm{mg} / \mathrm{L}$. Additional work-up to exclude autoimmune, nutritional, and inflammatory causes of bilateral vision loss, including complete blood count with platelet count, antinuclear antibody (ANA), antibody markers for neuromyelitis optica, Lyme disease and syphilis, serum levels of vitamin B12 and folic acid, and chest CT were normal or negative. She was diagnosed with bilateral retrobulbar optic neuritis and treated with 5 days of 1 gram IV methylprednisolone with a tapering dose of oral prednisone starting at $60 \mathrm{mg} /$ day.

She presented to our clinic 3 weeks after onset of visual loss. On ophthalmic examination, visual acuity was no light
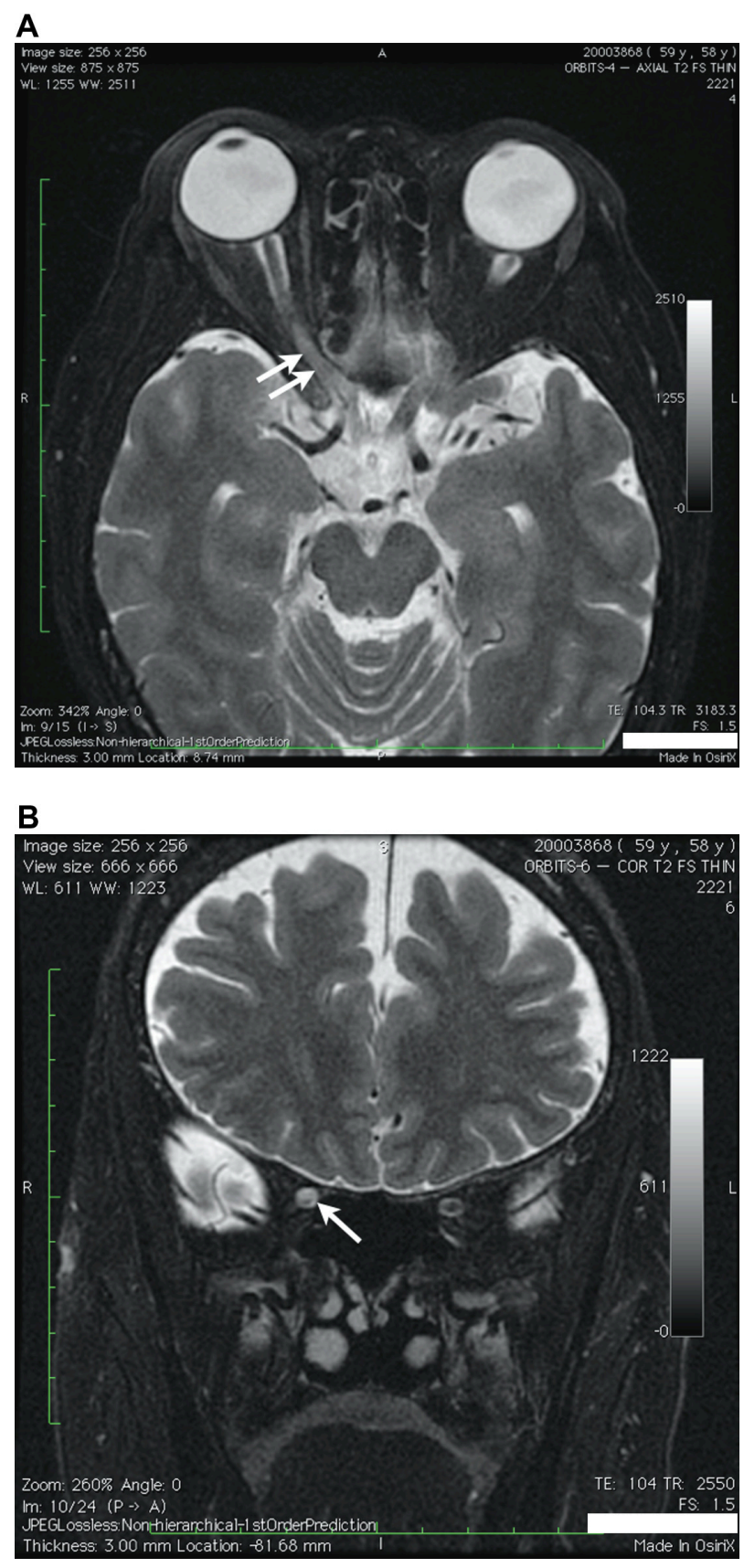

Figure I Orbital MRI T2-weighted sequences demonstrating hyperintensity in the posterior aspect of the right optic nerve (arrows). Hyperintensity is seen in both axial (A) and coronal (B) images.

Abbreviation: MRI, magnetic resonance imaging.

perception in each eye. The pupils were 6 millimeters and nonreactive to light. They constricted on convergence effort. Extraocular muscle movements were full. Anterior chambers were deep and quiet. She had mild nuclear sclerotic cataracts in both eyes; intraocular pressures were normal. The right optic nerve was swollen, while the left optic nerve was tilted with peripapillary atrophy (Figure 2). Both optic nerve heads were hyperemic. The retinal vasculature, especially the 


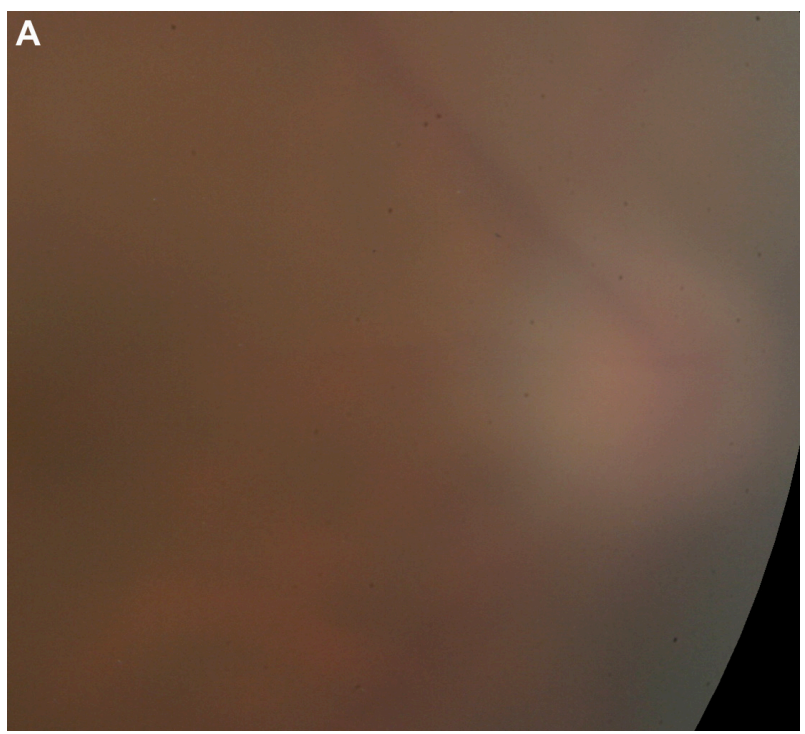

B

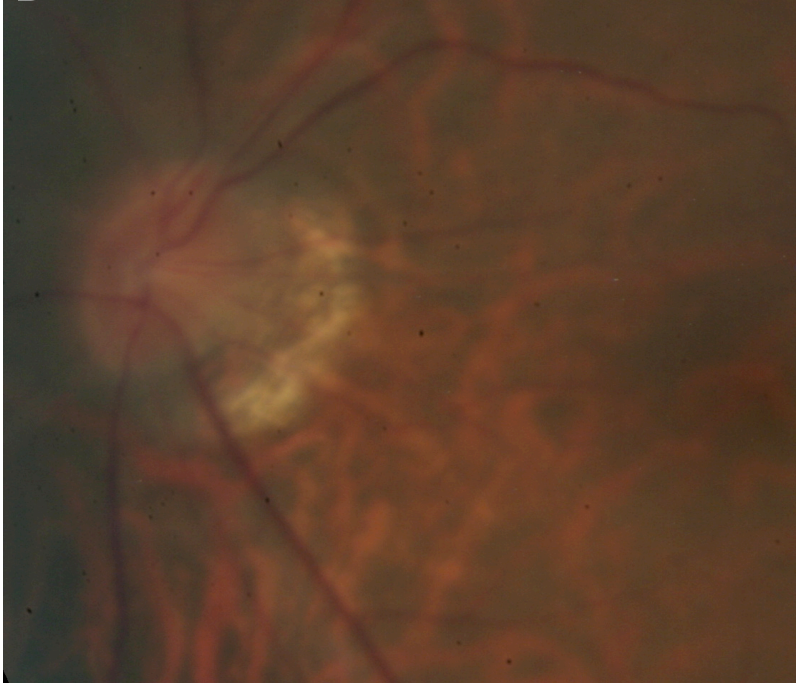

Figure 2 Fundus photos at presentation. (A) optic disc swelling in the right eye and (B) a tilted optic disc with peripapillary atrophy in the left eye.

arterioles, was markedly attenuated in both eyes. There was an inactive chorioretinal scar anterior to the inferior arcade in the right eye. No retinal hemorrhages, exudates, or cotton wool spots were appreciated.

Fluorescein angiogram of the right fundus showed delayed filling of the choroid and retina and late dye leakage from the optic disc. Late images of the left fundus did not show any abnormal leakage, staining, or capillary nonperfusion. No further treatment was recommended aside from psychiatric consultation, which the patient refused.

She returned after 8 weeks. Repeat examination revealed vision remained to be no light perception. There was diffuse optic nerve pallor of the right eye more than

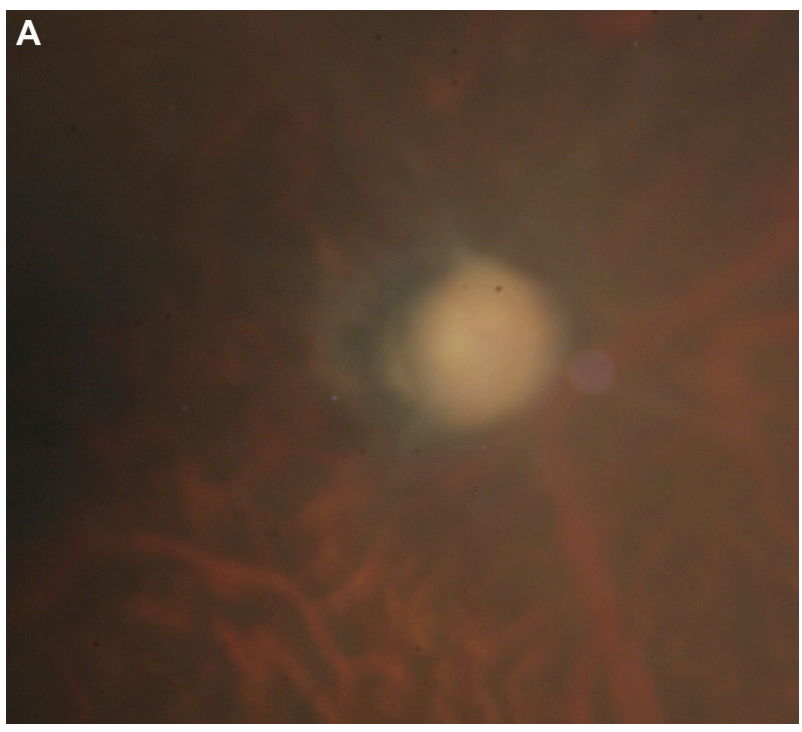

B

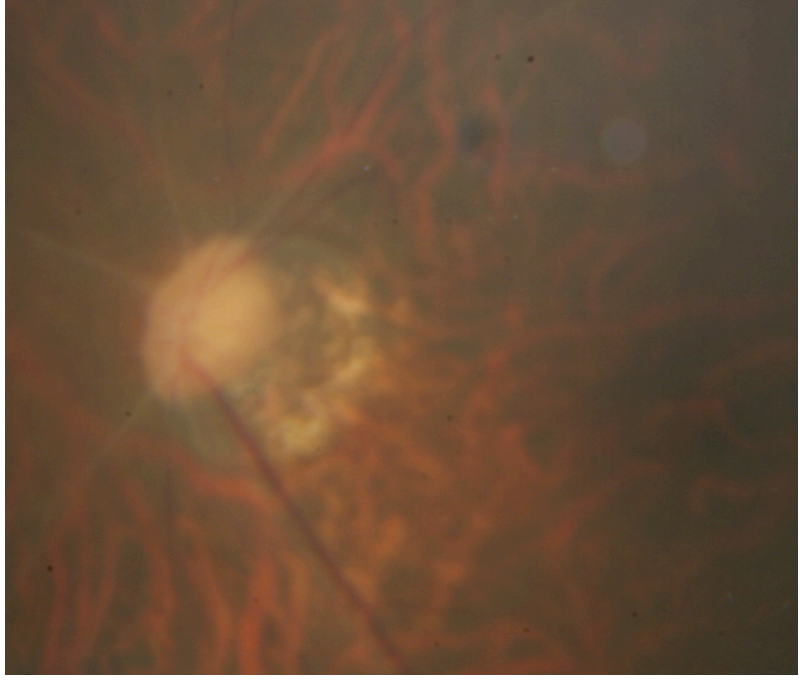

Figure 3 Optic disc appearance about 3 months after radiofrequency radiation injury. Diffuse optic disc pallor is shown in both eyes with $(\mathbf{A})$ resolving right optic disc swelling and (B) marked arteriolar attenuation.

the left (Figure 3). The retina exhibited marked arteriolar narrowing. On 3 month follow-up, the patient's vision loss was unchanged and there were no significant changes to her health. She continued to deny any systemic symptoms.

\section{Discussion}

We present a patient with subacute, painless, binocular, complete vision loss after orbital RFR exposure. Disc swelling with dye leakage on fluorescein angiography was present in the right eye, while the left eye had a normal-appearing disc. Gadolinium-enhancement and T2 hyperintensity of the optic nerves were appreciated on the MRI. 
Sudden monocular blindness has been reported as a result of trigeminal RF rhizotomy in the treatment of trigeminal neuralgia. ${ }^{2,3}$ In these cases optic nerve damage resulted from misdirection of the stylet to the inferior orbital fissure and into the optic canal where it was in contact with the optic nerve. In three of four reported cases wherein immediate ophthalmologic referral and assessment were made, fundoscopy revealed normal-appearing optic discs. Pathology of the optic nerve from one of these cases revealed edematous axonal fibers and coagulation necrosis with focal areas of acute hemorrhage compatible with thermal injury to the optic nerve $2-3 \mathrm{~cm}$ behind the globe. ${ }^{3}$

Irreversibility of vision loss in our patient suggests that there was axonal injury rather than pure demyelination, just as was seen in the rhizotomy cases. A purely demyelinating process may be reversible. Electrocautery can cause variable degrees of demyelination. ${ }^{5}$ In a case report, it was shown that blindness from electrocautery use during orbital surgery was reversible, with the patient regaining 20/40 vision from no light perception 10 weeks after surgery.

While the case reports we have mentioned involved direct contact of the optic nerve with the RF-generating instrument, RF energy can penetrate deeper into the body and cause heating of remote tissues or organs. ${ }^{1}$ For example, monocular blindness has been reported following electrocoagulation during sinus surgery, ${ }^{4}$ although even in this case the instrument was directly adjacent to the optic canal. In our case, the RF source was between 5 and $25 \mathrm{~mm}$ from the injured tissue (retinal ganglion cell layer), which is quite different from previously reported cases. We thus postulate that thermal injury can occur without direct contact with the instrument, which is likely what happened to the patient we have reported as the RF application occurred on the eyelids. Thermal injury to the retinal ganglion cells might occur though disruption of axonal ultrastructure, including microtubules, ${ }^{7}$ which would result in the subacute vision loss noted by this patient. Direct thermal injury and occlusion of the retinal and/or optic nerve vasculature is much less likely to have occurred because immediate severe vision loss would be expected in that case.

Other possible causes of this patient's vision loss include Leber's hereditary optic neuropathy (LHON), which can cause subacute, simultaneous, painless, bilateral, profound vision loss but rarely gives no light perception. Although LHON is more common in men, the rate of vision loss among women at risk ranges from $8 \%$ to $32 \%{ }^{8}$ LHON in the acute stage is also characterized by the triad of fundus findings, including pseudoedema of the peripapillary nerve fiber layer, circumpapillary telangiectatic microangiopathy, and absence of disc leakage on fluorescein angiography. True optic disc edema with documentation of optic disc leakage on fluorescein angiography in our patient points against LHON as the diagnosis.

Although arteritic ischemic optic neuropathy (AION) from giant cell arteritis (GCA) has been reported to be associated with optic nerve enhancement with gadolinium on MRI, ${ }^{9}$ vision loss from bilateral simultaneous AION was unlikely due to absence of other systemic symptoms of GCA. The optic discs did not exhibit the typical pallid swelling of AION. ESR, CRP, and platelet count were not elevated, and the patient's age also makes GCA less likely.

Neuromyelitis optica (NMO) may cause bilateral, simultaneous, severe optic neuritis with poor visual recovery. While a negative NMO immunoglobulin $\mathrm{G}$ (IgG) test does not rule out NMO, our patient fails to meet the diagnostic criteria for NMO, which requires not only optic neuritis but also transverse myelitis plus two of three of the following: NMO IgG seropositivity, longitudinally extensive transverse myelitis on imaging, and a brain MRI not suggestive of multiple sclerosis. ${ }^{10}$

Nutritional optic neuropathy is usually characterized by slowly progressive, bilateral visual decline. Total vision loss is also unusual in nutritional optic neuropathy. ${ }^{11}$ Normal vitamin B12 and folate levels further exclude nutritional optic neuropathy as the cause of our patient's vision loss. Other causes of bilateral optic neuritis, including sarcoidosis, syphilis, and Lyme disease, were excluded by negative laboratory test results. Optic neuropathy in these cases is typically associated with pain and may show a dramatic response to steroids. Infiltrative optic neuropathy, particularly meningeal carcinomatosis, may cause subacute, profound, bilateral vision loss. However, this seems unlikely due to the absence of other neurological signs or MRI findings.

Visual loss by thermal injury following RF exposure is rare. Without tissue analysis, the diagnosis of RFR-induced optic neuropathy is made by exclusion of other entities. We encourage physicians to inquire about RFR-generating device use in patients with optic neuropathy in whom the cause of the vision loss may be elusive.

\section{Acknowledgments}

This research was supported in part by an unrestricted grant to the Wilmer Eye Institute from Research to Prevent Blindness, Inc, New York, NY, USA.

\section{Disclosure}

The authors report no conflict of interest in this work. 


\section{References}

1. IEEE Committee on Man and Radiation. Medical aspects of radiofrequency radiation overexposure. Health Phys. 2002;82(3):387-391.

2. Feiler V, Godel V, Lazar M. Sudden blindness after thermocoagulation of the trigeminal ganglion. Ann Ophthalmol. 1990;22:339-340.

3. Egan RA, Pless M, Shults WT. Monocular blindness as a complication of trigeminal radiofrequency rhizotomy. Am J Ophthalmol. 2001;131:237-240.

4. Vanden Abeele D, Clemens, A, Tassignon MJ, van de Heyning PH. Blindness due to electrocoagulation following functional endoscopic sinus surgery. J Laryngol Otol. 1996;110:261-264.

5. Schietroma JJ, Tenzel RR. The effects of cautery on the optic nerve. Ophthalmic Plast Reconstr Surg. 1990;6(2):102-107.

6. Elder JA. Ocular effects of radiofrequency energy. Bioelectromagnetics. 2003;Suppl 6:S148-S161.
7. Erdine S, Bilir A, Cosman ER. Ultrastructural changes to axons following exposure to pulsed radiofrequency fields. Pain Pract. 2009;9:407-417.

8. Newman NJ. Leber's hereditary optic neuropathy. Arch Neurol. 1993; 50:540-548.

9. Lee AG, Eggenberger ER, Kaufman Di, Manrique C. Optic nerve enhancement on magnetic resonance imaging in arteritic ischemic optic neuropathy. J Neuroophthalmol. 1999;19:235-237.

10. Thornton IL, Rizzo JF, Cestari DM. Neuromyelitis optica: a review. Semin Ophthalmol. 2011;26(4-5):337-341.

11. Phillips PH. Toxic and deficiency optic neuropathies. In: Miller NR, Newan NJ, Biousse V, Kerrison JB, editors. Walsh and Hoyt's Clinical Neuro-Ophthalmology. 6th edition. Philadelphia: Lippincott Williams and Wilkins; 2005:447-463.
Clinical Ophthalmology

\section{Publish your work in this journal}

Clinical Ophthalmology is an international, peer-reviewed journal covering all subspecialties within ophthalmology. Key topics include: Optometry; Visual science; Pharmacology and drug therapy in eye diseases; Basic Sciences; Primary and Secondary eye care; Patien Safety and Quality of Care Improvements. This journal is indexed on

Submit your manuscript here: http://www.dovepress.com/clinical-ophthalmology-journal

\section{Dovepress}

PubMed Central and CAS, and is the official journal of The Society of Clinical Ophthalmology (SCO). The manuscript management system is completely online and includes a very quick and fair peer-review system, which is all easy to use. Visit http://www.dovepress.com/ testimonials.php to read real quotes from published authors. 